\title{
Isoniazid Induced Psychosis: A Case Report
}

\author{
Dr. Natraj $\mathbf{M}^{1 *}$, Dr. Gulnar Begum ${ }^{1}$, Dr. S. P. Burma ${ }^{1}$
}

\author{
${ }^{1}$ Department of Chest \& TB, Andaman and Nicobar Islands Institute of Medical Sciences, Port Blair, Andaman and Nicobar Islands \\ DOI: $10.36347 /$ sjmcr.2021.v09i03.022 \\ | Received: 27.02.2021 | Accepted: 15.03.2021 | Published: 21.03.2021 \\ *Corresponding author: Dr. Natraj M

\section{Abstract} \\ Isoniazid is a first line drug used in the treatment of tuberculosis. Psychosis is a state of altered mentation and \\ behavior. Although psychosis is known complication of isoniazid therapy, type and onset of symptoms is highly \\ variable. Here we report a case of 44 years old female who developed psychosis characterized by irrelevant talks, \\ agitation, and echolia after four days of starting ATT. \\ Keywords: Secondary psychosis, isoniazid, tuberculosis, adverse drug reaction. \\ Copyright () 2021 The Author(s): This is an open-access article distributed under the terms of the Creative Commons Attribution 4.0 International \\ License (CC BY-NC 4.0) which permits unrestricted use, distribution, and reproduction in any medium for non-commercial use provided the original \\ author and source are credited.
}

\section{INTRODUCTION}

Tuberculosis continues to be a challenge and an important public health problem $[1,2]$. Drugs used in the treatment of drug sensitive include rifampicin, isoniazid, ethambutol and pyrazinamide [3]. Isoniazid was first discovered in 1912 but was introduced only in 1952 by Robitzek [4]. Adverse drug reactions for isoniazid include hepatitis, peripheral neuropathy, abdominal symptoms like nausea and vomiting, skin rashes, lupus like syndromes and central nervous system effects like seizures, irritability and psychosis [3].

\section{Case Report}

A 44 years old married female, weighing 50 kgs presented to Department of Chest \& TB with complaints of dry cough, shortness of breath on excertion, evening rise of temperature, right pleuritic pain and loss of apatite. She had no history of any co morbids, significant past medical or surgical history or substance abuse. On auscultation air entry was decreased on the right hemithorax with chest x-ray showing right pleural effusion. Thoracocentesis was performed and about $700 \mathrm{ml}$ of straw colored fluid was aspirated and sent for analysis. With pleural fluid reports suggestive of tuberculosis patient was started on ATT according to her weight (4 FDC 4 Tablets/Day). Her hematological investigations were within normal level.

Patient along with her attender presented back to our department after ten days with complaints of irrelevant talks, acute agitation, loss of sleep and echolalia. A psychiatric opinion was obtained and her acute agitation was managed with lorazepam. There was no neurological deficit and her CT brain was essentially normal. Her hematologic and CSF analysis was also found to be within normal limits. So provisional diagnosis of drug-induced psychosis were considered.

Patient was then started on modified ATT without containing isoniazid. She became completely free of psychotic symptoms over the next three days and her anti psychotic medication was slowly weaned and stopped. Considering the importance of isoniazid consent was sought from the patient to restart isoniazid in a small dose. Patient and her family refused to take isoniazid, so she was discharged with modified ATT and serial chest $\mathrm{x}$-ray shows significant radiological improvement. There were no further psychiatric manifestations.

\section{DisCUSSION}

Jackson first highlighted the first description of isoniazid causing psychosis in the year 1957 [5]. Since then there has been many case reports showing the association between isoniazid and psychosis [6]. Two main mechanisms has been postulated for isoniazid causing psychosis, (A) depletion of pyridoxine and (B) inhibition of monoamine oxidase leading to changes in levels of serotonin and catecholamine [7]. According to various case reports the risk factors for isoniazid causing psychosis includes old age, personal and family history of psychological illness, alcohol intake, diabetics, malnutrition, hepatocellular insufficiency and hyperthyroidism [4]. Another possible reason may be 
due to the pharmacokinetic property of isoniazid. Usually the drug gets rapidly absorbed from GI tract within one to two hours of ingestion. But it is estimated that $40 \%$ of the Indian population are slow acetylators leading to drug accumulation and more side effects [1]. Usually the onset of psychiatric symptoms varies from days to months, but usually seen after few weeks of starting of isoniazid [5]. Various psychiatric symptoms has been documented in the literature including paranoid delusions, hallucinations (auditory, visual and tactile), suicide tendencies, mood symptoms, anxiety, loss of sleep, twitchings, disorientation, loosening of associations and echolalia $[8,9]$.

The usual approach in treating patients with isoniazid induced psychosis includes withdrawing the drug first, treating the psychosis and then to gradually reintroduce at a lower dosage one the psychosis completely settles [10]. Some authors have also suggested restarting isoniazid with higher doses of pyridoxine while others have recommended using antipsychotic drugs along with high doses of pyridoxine [11]. But since our patient did not give consent for restating isoniazid she was started on modified anti tuberculous drug.

\section{Conclusion}

Tuberculosis is one of the most common infectious diseases in India. Isoniazid being one of the most commonly used drugs due to its potency, physicians must be aware of all the possible side effects it can cause.

\section{REFERENCES}

1. Arya S, Sukhija G, Singh H. Acute psychosis after recent isoniazid initiation. Journal of clinical and diagnostic research: JCDR. 2015 Jun;9(6):VD01.
2. Kaur T, Kaur K, Malhotra P. Isoniazid Induced Psychosis: A Case Report. Journal of Nepal Paediatric Society, 2019;39(3), 189-192.

3. Forget EJ, Menzies D. Adverse reactions to firstline antituberculosis drugs. Expert Opin Drug Saf. 2006;5(2):23149. doi:10.1517/14740338.5.2.2 31

4. Menon VB, Shaji R, Pereira P, Chikkaveeraiah SK, Ramesh M. Isoniazid associated psychosis: a case series of 3 patients. Indian Journal of Pharmacy Practice. 2017 Apr;10(2):142-6.

5. Jackson SL. Psychosis due to isoniazid. Br Med J. 1957;28:743-

5. doi.org/10.1136/bmj.2.5047.743

6. Witkowski A, Manabat C, Bourgeois J. Isoniazidassociated psychosis. Gen Hosp Psychiatry. 2007;29(1):856. doi:10.1016/j.genhosp psych.2006.10.010

7. Pallone KA, Goldman MP, Fuller MA. Isoniazidassociated psychosis: case report and review of the literature. Ann Pharmacother. 1993;27(2):167-70.

8. Herrag M, Sajiai H, Alaoui Yazidi A. Acute psychotic access following the administration of isoniazid. Rev Pneumol Clin. 2011;67(2):118 20. doi: 10.1016/j.pneumo.2010.03.002

9. Masood I, Bhat S, Beigh A, Gupta V. Isoniazidinduced psychosis in a patient on DOTS therapy. Annals of Tropical Medicine and Public Health. 2011;4(2):126-7

10. Oninla SO, Oyedeji GA, Oninla OA, GbadeboAina. Isoniazid induced psychosis in two children treated for tuberculosis: case reports and literature review. IJMPCR. 2016;6(4):1-6.

11. Isoniazid induced psychosis- a clinical dilemma. $\mathbf{J}$ Indian Acad Clin Med. 2002;3(3):306-7. 